\title{
Metabolism of conjugated sterols in eggplant. Part 2. Phospholipid : steryl glucoside acyltransferase
}

\author{
Anna Potocka and Jan Zimowski ${ }^{\bowtie}$ \\ Institute of Biochemistry, Warsaw University, Warszawa, Poland
}

Received: 05 March, 2007; revised: 09 November, 2007; accepted: 13 December, 2007

available on-line: 16 January, 2008

\begin{abstract}
A membrane-bound phospholipid: steryl glucoside acyltransferase from Solanum melongena leaves was partially purified and its specificity and molecular as well as kinetic properties were defined. Among the steryl glycosides tested (e.g. typical plant steryl glucosides, steryl galactosides and cholesteryl xyloside) the highest activity was found with cholesteryl glucoside, but some structurally related compounds such as sito- and stigmasteryl glucoside or galactoside as well as cholesteryl galactoside were also acylated, albeit at lower rates. The investigated enzyme was able to use all classes of phosphoglycerolipids (phosphatidylethanolamine, phosphatidylcholine, phosphatidylserine, phosphatidylinositol, phosphatidylglycerol) as an acyl source for biosynthesis of acylated steryl glucoside. Among them 1,2-dimirystoylphosphatidylic acid appeared to be the best acyl donor. Apart from phosphoglycerolipids, 1,2-diacylglycerols were also used as acyl donor for steryl glucoside acylation, although at a distinctly lower rate. The acyl moiety was transferred from the C-1 position of phospholipid molecule. The investigated acyltrasferase activity was stimulated by 2-mercaptoethanol, Triton X-100, 1-monoacylglycerols and inhibited in the presence of divalent cations such as $\mathrm{Ca}^{2+}, \mathrm{Mn}^{2+}, \mathrm{Zn}^{2+}$ or $\mathrm{Co}^{2+}$, some lipids (MDGD, ceramide), detergents (Tween 20, 40, 60 and 80, Tyloxapol, sodium deoxycholate) and high ionic strength.
\end{abstract}

Keywords: acylated steryl glucoside, phospholipid : steryl glucoside acyltransferase, Solanum melongena, steryl glucoside

\section{INTRODUCTION}

Apart from steryl glucosides, steryl esters and free sterols, acylated steryl glucosides (ASG) are common components of plant membranes (Wojciechowski, 1991). The molecular heterogeneity of ASG is a result of the complex composition of both sterol and fatty acid constituents. In general, the sterol profiles of ASG fractions from vascular plants are more or less similar to those of the free sterol fractions and usually contain a number of typical saturated and unsaturated fatty acids (mainly $\mathrm{C}_{16}$ and $\mathrm{C}_{18}$ ) (Lepage, 1964; Galliard, 1968; Staphylakis \& Gegiou, 1985; Kojima et al., 1989; Kintia \& Wojciechowski,
1974; Wojciechowski \& Zimowski, 1975; Khan et al., 1997).

Whether ASG are active metabolic compounds or not is an unsolved problem. Some hypotheses consider these conjugates to be final products of sterol metabolism, others suggest that processes of acylation or deacylation of SG or ASG, respectively, incorporated into membranes can play a significant role in the properties of these membranes or in modulation of membraneous enzyme activity. For instance, it has been shown that SG acylation inhibits the activity of phospholipid-induced $\mathrm{H}^{+}$-ATPase from tonoplast of cultured rice cells (Yamaguchi \& Kasamo, 2001). On the other hand, some stress

Corresponding author: Jan Zimowski, Instytut Biochemii UW, I. Miecznikowa 1, 02-096 Warszawa, Poland; phone: (48) 22 554 3404; fax: (48) 22554 3221; e-mail: janjzim@biol.uw.edu.pl

Abbreviations: ASG, acyl steryl glucosides; ATase(s), acyltransferase(s); DGDG, digalactosyldiacylglycerols; MGDG, monogalactosyldiacylglycerols; NEM, N-ethylmaleimide; PA, phosphatidic acid; PC, phosphatidylcholine; pCMBS, $p$-chloromercuribenzenesulfonic acid; PE, phosphatidylethanolamine; PG, phosphatidylglycerol; PI, phosphatidylinositol; PL(s), phospholipid(s); PS, phosphatidylserine; SG, steryl glucosides. 
factors, e.g. significant variation of temperature, the presence of heavy metals (e.g. $\mathrm{Ag}^{+}, \mathrm{Cu}^{2+}$ and $\mathrm{Ca}^{2+}$ ions) or fungal cellulase or xylanase, gamma-irradiation or $\mathrm{O}_{3}$ treatment generated changes in the contents of ASG in plant membranes (Whitaker et al., 1990; Palta et al., 1993; Moreau et al., 1994; Żelazny et al., 1995; Picchioni et al., 1996; 1998; McDonald et al., 2000; Quartacci et al., 2001). These data strongly suggest that metabolism of conjugated forms of sterols is involved in plant response to biotic or abiotic stress. Apart from the physiological role of ASG mentioned above, these compounds have same pharmacological activity. Some data show that ASG from Momordica charantia (Guevara et al., 1990) and soybean (Kiriakidis et al., 1997) have antimutagenic and antiproliferative activity, respectively, and ASG from Polygonum hydropiper have a toxic effect on earthworms (Chaudhuri et al., 1996).

However, there are still only a few data about ASG biosynthesis. It has been shown that ASG are formed in the presence of SG and PL or galactoglycerides (Wojciechowski \& Zimowski, 1975; Heinz et al., 1975; Eichenberger \& Siegrist 1975; Forsee et al., 1976). However, contradictory results were presented regarding the acyl donor specificity of the enzyme from different sources. An enzyme preparation from Calendula officinalis seedlings catalyzed acyl transfer only from PL (Wojciechowski \& Zimowski, 1975). Acyltransferase from broad bean leaves (Heinz et al., 1975) used only galactoglycerides, while the enzyme from carrot roots (Eichenberger \& Siegrist, 1975) utilized both (i.e. PL and galactolipids).

We now report the isolation of a completely delipidated enzyme preparation of membraneous acyltransferase catalyzing formation of ASG from eggplant seedlings. We also present the molecular and kinetic properties and specificity of this enzyme.

\section{MATERIAL AND METHODS}

Plant material. Eggplant (Solanum melongena L., cv Black Beauty) were grown on artificial support (Perlite) in a greenhouse at a $16 / 8 \mathrm{~h}$ photoperiod, at $25 / 20^{\circ} \mathrm{C}$ day/night. Leaves were cut off from 9 -weeks old plants.

Enzyme preparation. Fresh leaves (100 g of fresh mass) were homogenized with $200 \mathrm{ml}$ of icecold $0.1 \mathrm{M}$ Tris/ $\mathrm{HCl}$ buffer, $\mathrm{pH}$ 7.3, containing 10 $\mathrm{mM}$ 2-mercaptoethanol (buffer A). The homogenate was filtered through cheesecloth and centrifuged at $20000 \times g(20 \mathrm{~min})$. The $20000 \times g$ pellet (the membrane fraction) was resuspended in buffer $\mathrm{A}$ and added dropwise to a 10 -fold volume of cold $\left(-20^{\circ} \mathrm{C}\right)$ acetone so "acetone powder I" was obtained as previously described (Zimowski, 1991). "Acetone powder I" was resuspended in buffer A containing $0.1 \%$ Triton X-100 and centrifuged at $20000 \times g(20$ min). Supernatant was added dropwise to a 10 -fold volume of acetone $\left(4^{\circ} \mathrm{C}\right)$, and "acetone powder II" were obtained as previously described (Zimowski, 1991). "Acetone powder II" was resuspended in $0.1 \mathrm{M}$ phosphate buffer, $\mathrm{pH}$ 6.5, containing $0.05 \%$ Triton X-100 and $10 \mathrm{mM}$ 2-mercaptoethanol (buffer $B)$, to give completely delipidated enzyme preparation.

Acyltransferase assays. The standard reaction mixture contained in a total volume of $0.52 \mathrm{ml}$ : $0.1 \mathrm{mg}$ of "acetone powder II" in $0.5 \mathrm{ml}$ of buffer B; 0.4 nmol of $\left[4{ }^{-14} \mathrm{C}\right]$ cholesteryl glucoside $\left(5.0 \times 10^{4}\right.$ $\mathrm{dpm}$ ) or $10 \mathrm{nmol}$ of steryl glycoside in $0.01 \mathrm{ml}$ of ethanol, and acyl residue donor $(10 \mathrm{nmol})$ or labelled acyl donor $\left(10^{5} \mathrm{dpm} ; 0.9 \mathrm{nmol}\right)$ in $0.01 \mathrm{ml}$ of ethanol. Once the enzyme preparation was added, the reaction was run at $35^{\circ} \mathrm{C}$ for $30 \mathrm{~min}$ and then stopped by adding $1 \mathrm{ml}$ methanol and heating for 3 min in a boiling water bath. Subsequently samples were extracted 3 times with $4 \mathrm{ml}$ of 1-butanol saturated with water and collected butanolic extracts were washed several times with water saturated with 1-butanol. The samples were air-dried and applied on silica gel plates and developed with chloroform/methanol $(9: 1, \mathrm{v} / \mathrm{v})$, as a solvent. Labelled compounds were localized by autoradiography and their chromatographic mobilities were compared with those of the following authentic reference compounds: 6'-O-palmitoyl 3 3 -O-D-monoglucopyranoside of cholesterol or sitosterol $\left(R_{F}=0.54\right)$ and 6'-O-palmitoyl 33-O-D-monogalactopyranoside of cholesterol or sitosterol $\left(R_{F}=0.51\right)$. The radioactivity measurements were carried out as previously described (Zimowski, 1991).

Other methods. Unlabelled steroidal monoglycosides i.e. chole-, sito- or stigmasteryl 3-O- $\beta$ D-monoglucopyranoside or 3-O- $\beta$-D-monogalactopyranoside were obtained as previously described (Janiszowska et al., 1980).

Unlabelled acyl steroidal monoglucosides i.e. 6'-O-palmitoyl-, 6'-O-oleyl- or 6'-O-linoeyl deriva-

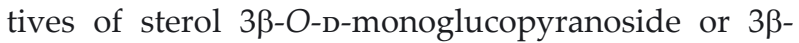
$\mathrm{O}$-D-monogalactopyranoside were obtained as previously described (Kiribuchi et al., 1967).

Gel filtration was carried out on a Sephadex G-150 column $(2 \times 40 \mathrm{~cm})$ equilibrated with buffer B.

Triton X-100 removed on a Bio-Beads SM-2 column $(1 \times 5 \mathrm{~cm})$ equilibrated with $0.1 \mathrm{M}$ phosphate buffer, $\mathrm{pH}$ 6.5, containing $10 \mathrm{mM}$ 2-mercaptoethanol (buffer C). The completely delipidated enzyme preparation (10 mg protein in $1 \mathrm{ml}$ of buffer B) was applied onto the column and eluted with $15 \mathrm{ml}$ of buffer $\mathrm{C}$ at a flow rate of $0.4 \mathrm{ml} / \mathrm{min}$ and acyltransferase activity was assayed. 


\section{RESULTS AND DISCUSSION}

Preliminary incubations performed in the presence of $\left[{ }^{14} \mathrm{C}\right]$ cholesteryl glucoside with a homogenate obtained from 9-week-old S. melongena leaves indicated that this enzyme preparation was able to catalyze the formation of one radioactive product only. This product had the same chromatographical mobility as ASG fraction isolated from eggplant

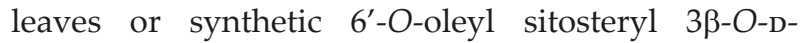
monoglucopyranoside.

Similar incubations with subcellular fractions separated by differential centrifugation indicated that almost whole ATase activity was connected with particulate fractions. The fraction sedimenting at $3000 \times g$ was the most active, exhibiting $57 \%$ of the total enzyme activity, so it seems that eggplant ATase activity is strongly bound with the cell membrane. It was solubilized only in the presence of Triton X-100 (complete solubilization was observed with $0.1 \%$ concentration of this detergent). Similar, membraneous localization of ATase activity was observed for the enzyme from Calendula officinalis leaves (Wojciechowski \& Zimowski, 1975), Solanum tuberosum amyloplast membranes (Catz et al., 1985), Avena sativa seedlings (Misiak et al., 1991), cotton fibers (Forsee et al., 1976), spinach leaves (Heinz et al., 1978); however, the enzyme from carrot roots (Eichenberger \& Siegrist, 1975) and Vicia faba leaves (Heinz et al., 1975) are localized in the cytosolic fraction. Additionally, it seems that all these membraneous ATases co-occur in the same structures with UDPglucose : sterol glucosyltransferase (Wojciechowski, 1991).

In contrast to native enzyme preparations from eggplant and the enzymes isolated from other plants mentioned above, which were able to synthesize acylated steryl glucoside in the absence of exogenous acyl donor or acceptor, the completely delipidated enzyme preparation from eggplant exhibited a complete loss of the ability to synthesize acyl steryl glucoside when labelled steryl glucoside or full lipid fraction was given alone as the acyl residue acceptor or acyl residue donor, respectively.

\section{Molecular and kinetic properties of acyltransferase from eggplant leaves}

The enzyme exhibits an apparent $K_{\mathrm{m}}=16 \mu \mathrm{M}$ for cholesteryl monoglucoside and $18.2 \mu \mathrm{M}$ for dimyristoylphosphatidic acid. Similar $K_{m}$ values for a mixture of natural steryl glucosides and phosphatidylethanolamine were reported for ATase from cotton fibers (70 and $30 \mu \mathrm{M}$, respectively) (Forsee et al., 1976).

Maximal stimulation of steryl glucoside acylation was observed in the presence of $0.5 \%$ Triton X-
100 (about 32-fold) but other investigated detergents (e.g. Tween 20, 40, 60 and 80, Tyloxapol and deoxycholic acid sodium salt) had a strong inhibitory effect. The removal of Triton X-100 from incubation mixture by filtration on Bio-Beads SM-2 caused a loss of $95 \%$ of ATase activity. Addition of this detergent restored ASG formation (in 60\%). This strongly suggests that Triton X-100 is necessary for eggplant ATase activity. A stimulatory effect of Triton X-100 was also observed for acyltransferases from cotton fibers (Forsee et al., 1976), Calendula officinalis leaves (Wojciechowski \& Zimowski, 1975) and Solanum tuberosum amyloplast membranes (Catz et al., 1985).

The formation of ASG by the eggplant enzyme was stimulated by 2 -mercaptoethanol (76\% stimulation) and strongly inhibited in the presence of some divalent metal ions, such as $\mathrm{Ca}^{2+}, \mathrm{Mn}^{2+}, \mathrm{Co}^{2+}, \mathrm{Zn}^{2+}$ $\left(\mathrm{I}_{50}=0.57,0.63,0.83\right.$ and $0.08 \mathrm{mM}$, respectively) and pCMBS and NEM $\left(\mathrm{I}_{50}=0.06\right.$ and $2.4 \mathrm{mM}$, respectively) whereas $\mathrm{Mg}^{2+}$ appeared to have no effect on ATase activity. EDTA slightly stimulated formation of ASG (34\% stimulation). The inhibitory effect of $\mathrm{Ca}^{2+}$ on ASG biosynthesis may be a result of formation of insoluble calcium salt of PL.

The influence of some potential ATase effectors has been studied only for maturing pea seeds (Baisted, 1978). Similar to the eggplant enzyme, the pea ATase was strongly inhibited by $p$ CMBS and NEM as well as $\mathrm{Zn}^{2+}$ ion. However, in opposition to the ATase from eggplant, ATase activity from mature pea seeds was activated by some divalent ions, i.e. $\mathrm{Ca}^{2+}$ and $\mathrm{Mg}^{2+}$, and inhibited by EDTA. These results may suggest that the investigated enzymes, i.e. ATase from eggplant and the pea enzyme require reduced cysteine residues for their full activity. On the other hand, eggplant ATase, in contrast to the pea enzyme, has no requirement for metal cofactors for acylation of steryl glucoside.

Acyl steryl glucoside synthesis was also sensitive to increased ionic strength of the incubation medium $\left(\mathrm{I}_{50}\right.$ for NaF was $\left.0.41 \mathrm{M}\right)$ and ethanol $\left(\mathrm{I}_{50}=\right.$ $2.6 \mathrm{M})$.

The apparent molecular mass of the eggplant ATase was established at $90 \mathrm{kDa}$. The molecular mass of enzymes which acylate steryl glucosides range from $60 \mathrm{kDa}$ for cytosolic carrot ATase (Eichenberger \& Siegrist, 1975) to $130 \mathrm{kDa}$ for the membraneous enzyme from Sinapis alba seedlings (Zimowski \& Wojciechowski, 1983).

Our data also indicate that an excess of all investigated steryl glycosides (e.g. unlabelled chole-, sito-, stigmasteryl glucoside or galactoside and cholesteryl xyloside) decreases the synthesis of labelled ASG in the presence of $\left[{ }^{14} \mathrm{C}\right]$ cholesteryl glucoside and 1,2-dimirystoyl-PA $\left(\mathrm{I}_{50}=0.005,0.013,0.014\right.$, $0.011,0.011,0.008$ and $0.062 \mathrm{mM}$, respectively). This could mean that all these steryl glycosides can com- 
pete for the active site of the enzyme with labelled cholesteryl glucoside and may act as potential acyl moiety acceptors. On the other hand, the about 10fold higher $I_{50}$ value for cholesteryl xyloside in comparison to cholesteryl glucoside strongly suggests that the presence of the $\mathrm{CH}_{2} \mathrm{OH}$ - group at $\mathrm{C}-6^{\prime}$ of the steryl glycoside moiety can play a very important role in the binding of steryl glucoside to the active site of eggplant ATase.

\section{Specificity of ATase from eggplant leaves towards acyl moiety donor and acceptor}

The completely delipidated enzyme preparation was used to study substrate specificity of the enzyme towards the acyl moiety donor in the presence of labelled cholesteryl monoglucoside. Synthetic and natural phospholipids, acylglycerols, acyl-CoA derivatives and glycolipids were tested as potential acyl donors. In all cases TLC analysis with subsequent autoradiography showed the presence of a single labelled product with chromatographic mobility expected for 6'-O-oleyl monoglucoside of cholesterol. Among the lipids tested (Table 1), 1,2dimirystoylphosphatidic acid and 1,2-dioleylphosphatidylglycerol were the best acyl donors (35.6 and 35.3 fkat, respectively). It is evident that the other investigated classes of phosphoglyceryllipids, e.g. PE, PC, PI and PS can be used as acyl moiety donors, however, at lower rates. The formation of ASG was also observed when 1,2-diacylglycerol, i.e. 1,2-dioleyl- or 1,2-dipalmitoylglycerol was used in the incubation mixture, however, with a 7- or 26-fold, respectively, lower rate comparing to 1,2dimirystoyl-PA. Steryl glucoside was practically not acylated in the presence of the other acylglycerols investigated (triacylglycerols and monoacylglycerols), sphingolipids (sphingomyeline), acylated CoA derivatives and glycolipids, e.g. MDGD and DGDG. It was found that ASG can be formed in the presence of phospho- or galactolipids as acyl moiety donors (Peaud-Lenoel, 1972; Wojciechowski \& Zimowski, 1975; Heinz et al., 1975; Eichenberger \& Siegrist, 1975; Forsee et al., 1976). Phosphoglycerides, particulary PE, were utilized for acylation of steryl glucoside by the enzyme from cotton fibers (Forsee et al., 1976). However, ATase from Vicia faba leaves was active only in the presence of chloroplast galactoglycerides (e.g. MDGD and DGDG) (Heinz et al., 1975), whereas the enzyme preparation from carrot roots (Eichenberger \& Siegrist, 1975) and mustard seedlings (Zimowski \& Wojciechowski, 1983) catalyzed acyl transfer from both galactoglycerides and phospholipids. This may suggest that in higher plants there are at least two distinct enzymes catalyzing the synthesis of ASG, i.e. one specific for phosphoglycerides and the second
- for galactoglycerides as acyl sources for SG acylation. However, it is also possible that these two acyl donors can be utilized for ASG formation by the same enzyme.

In order to establish whether the acylation rate of steryl glucoside depends on the acyl composition of the phospholipid molecule, several PA and PG synthetic molecular species containing fatty acids of different chain length or saturation were tested. Results presented in Table 1 clearly indicate that both fatty acid chain length and their saturation had a distinct effect on acyl steryl glucoside formation by ATase from eggplant leaves. The highest acylation rate was observed for the dimirystoyl-derivatives of both investigated PLs. The presence of an unsaturated fatty acid in the phospholipid molecule caused a 3-fold increase of steryl glucoside acylation rate comparing to saturated ones. These data strongly suggest that the rate of utilization of individual PLs as acyl donor may be determinated by its fatty acid composition.

To determine the specificity of the enzyme with respect to the acyl moiety acceptor, formation of labelled acyl steryl monoglycoside in the presence of 1,2-di-[ $\left[{ }^{14} \mathrm{C}\right]$ oleoyl-PC and various unlabelled steryl glycosides was tested. Our results clearly indicated that all steryl glucosides and galactosides tested (e.g. chole-, sito- and stigmasterol glycoside) were utilized as acyl acceptors. The acylation rate can be influenced by several structural factors such as the structure of the molecule fragment bound to the ring $\mathrm{D}$ of steroidal nucleus, conformation of the hydroxyl group at $\mathrm{C}-4^{\prime}$ or the presence of the $\mathrm{CH}_{2} \mathrm{OH}$ - group at $\mathrm{C}-5^{\prime}$ of sugar moiety. The highest radioactivity was incorporated to ASG when cholesteryl glucoside was present in the incubation mixture as an acyl acceptor (enzyme activity was 2.55 fkat). Acylated sito- and stigmasteryl glucoside was formated at a lower rate (about 66 and $83 \%$, respectively). The acylation rate for steryl glucoside epimers, e.g. chole-, sito- and stigmasteryl galactoside was about 2 -fold lower than for the respective glucosides. On the other hand, a steryl pentoside, i.e. cholesteryl xyloside, was not acylated by the investigated ATase.

It has been shown that the enzyme from cotton fibers is able to acylate sito-, stigma- and cholesteryl glucosides and that the best acyl acceptor is the second of them (Forsee et al., 1976). ATase from oat leaves catalyzed acylation of not only typical steryl glycosides but some of steroidal glucosides also, e.g. nuatigenin, pregnenolon and androstenolon glucoside (Kalinowska \& Wojciechowski, 1986; Misiak et al., 1991). Nuatigenin glucoside is an intermediate product in the biosynthesis of oat saponins, i.e. avenacosides, but its acylated form does not occur naturally in oat. 
Table 1. Specificity of ATase from S. melongena leaves towards acyl moiety donors.

Incubations were carried out in the presence of $\left[{ }^{14} \mathrm{C}\right]$ cholesteryl glucoside and acyl residue donor $(10 \mathrm{nmol})$. For other details see Material and Methods.

\begin{tabular}{|c|c|c|c|c|c|}
\hline \multirow[t]{2}{*}{ Acyl donor } & & & \multirow[t]{2}{*}{ Source } & \multicolumn{2}{|c|}{ Formation of acyl $\left[{ }^{14} \mathrm{C}\right]$ cholesteryl glucoside } \\
\hline & & & & fkat & Relative activity (\%) \\
\hline \multirow[t]{17}{*}{ Phospholipids } & \multirow{2}{*}{\multicolumn{2}{|c|}{$\begin{array}{l}\text { Phosphatidylethanolamine (PE) } \\
\text { Phosphatidylcholine (PC) }\end{array}$}} & soybean & 26.7 & 75.0 \\
\hline & & & soybean & 24.2 & 68.0 \\
\hline & & $\operatorname{diC}_{16: 0}$ & synthetic & 11.7 & 32.9 \\
\hline & \multirow{2}{*}{\multicolumn{2}{|c|}{ Phosphatidylinositol (PI) }} & soybean & 27.5 & 77.2 \\
\hline & & & bovine liver & 0.8 & 2.2 \\
\hline & \multirow[t]{2}{*}{ Phosphatidylserine (PS) } & & soybean & 18.6 & 52.2 \\
\hline & & $\operatorname{diC}_{16: 0}$ & synthetic & 8.1 & 22.7 \\
\hline & \multirow[t]{4}{*}{ Phosphatidylglycerol (PG) } & $\operatorname{diC}_{18: 1}$ & synthetic & 35.3 & 99.2 \\
\hline & & $\operatorname{diC}_{18: 0}$ & synthetic & 11.7 & 32.9 \\
\hline & & $\operatorname{diC}_{16: 0}$ & synthetic & 25.4 & 71.3 \\
\hline & & $\operatorname{diC}_{14: 0}^{10.0}$ & synthetic & 29.7 & 83.4 \\
\hline & \multirow[t]{5}{*}{ Phosphatidic acid (PA) } & $\operatorname{diC}_{18: 1}$ & synthetic & 25.6 & 71.9 \\
\hline & & $\operatorname{diC}_{16: 0}$ & synthetic & 4.4 & 12.4 \\
\hline & & $\operatorname{diC}_{14: 0}$ & synthetic & 35.6 & 100.0 \\
\hline & & $\operatorname{diC}_{12: 0}$ & synthetic & 25.6 & 71.9 \\
\hline & & $\operatorname{diC}_{10: 0}^{12: 0}$ & synthetic & 23.0 & 64.6 \\
\hline & \multicolumn{2}{|l|}{ Sphingomyeline } & bovine brain & 0.0 & 0.0 \\
\hline \multirow[t]{2}{*}{ Galactolipids } & \multicolumn{2}{|l|}{ Monogalactosyldiacylglycerol (MGDG) } & wheat & 0.0 & 0.0 \\
\hline & \multicolumn{2}{|l|}{ Digalactosyldiacylglycerol (DGDG) } & wheat & 0.0 & 0.0 \\
\hline \multirow[t]{3}{*}{ Acyl-CoA } & & $\mathrm{C}_{18: 1}$ & synthetic & 0.8 & 2.2 \\
\hline & & $\mathrm{C}_{14: 0}$ & synthetic & 0.6 & 1.7 \\
\hline & & $\mathrm{C}_{12: 0}$ & synthetic & 0.3 & 0.8 \\
\hline \multirow[t]{8}{*}{ Acylglycerols } & 1-Monooleoylglycerol & & synthetic & 0.0 & 0.0 \\
\hline & 1,2-Dioleoylglycerol & & synthetic & 5.3 & 14.9 \\
\hline & 1,3-Dioleoylglycerol & & synthetic & 5.6 & 15.7 \\
\hline & Trioleoylglycerol & & synthetic & 0.0 & 0.0 \\
\hline & 1-Monopalmitoylglycerol & & synthetic & 0.0 & 0.0 \\
\hline & 1,2-Dipalmitoylglycerol & & synthetic & 1.4 & 3.9 \\
\hline & 1,3-Dipalmitoylglycerol & & synthetic & 1.1 & 3.1 \\
\hline & Tripalmitoylglycerol & & synthetic & 0.0 & 0.0 \\
\hline
\end{tabular}

Our results suggest that the investigated ATase is rather specific for steryl glucoside as an acyl acceptor and PLs as acyl donor, therefore it may be regarded as a phospholipid : steryl glucoside acyltransferase.

To determine the position from which the acyl moiety of the PL molecule was transferred onto steryl glucoside, formation of $\left[{ }^{14} \mathrm{C}\right]$ oleoyl cholesteryl glucoside in the presence of 1,2-di$\left[{ }^{14} \mathrm{C}\right]$ oleoyl-PC or 1-palmitoyl-2- $\left[{ }^{14} \mathrm{C}\right]-$ oleoyl-PC and unlabelled cholesteryl glucoside was tested. Incorporation of radioactivity to $\left[{ }^{14} \mathrm{C}\right]$ oleoyl cholesteryl glucoside was observed only when 1,2-di$\left[{ }^{14} \mathrm{C}\right]$ oleoyl-PC was used as the acyl donor. This means that the acyl moiety is transferred onto steryl glucoside from the C-1 position of PL. However, the ATase from Vicia faba transferred the acyl moiety to steryl glucoside from the C-1 as well as the C-2 position of galactolipids, e.g. MGDG and DGDG (Heinz et al., 1975).

\section{Effect of some lipids on acylation of steryl gluco- side by ATase from eggplant}

Preliminary investigation showed that lipids (e.g. phospholipids, glycolipids and acylglycerols) had no effect on the biosynthesis of acylated sitosteryl glucoside by native enzyme preparation (i.e. homogenate, membraneous submolecular fractions). In contrast, sitosteryl glucoside acylation was distinctly modified in the presence of lipids when the completely delipidated enzyme preparation was used. An inhibitory effect on the formation of acyl steryl glucoside was observed in the presence of 1,3dioleoylglycerol, MGDG (from wheat) and ceramide from bovine brain $(87,53$ and $27 \%$ inhibition, re- 
spectively), whereas DGDG (from wheat) and trioleoylglycerol had no effect and 1-monooleoylglycerol slightly stimulated acyl steryl glucoside biosynthesis (about 20\% stimulation). The inhibition observed in the presence of 1,3-dioleoylglycerols or MGDG may be a result of competition with the acyl donor (e.g. 1,2-dimirystoyl-PA) for the active site of the enzyme. These results point to the possibility of in vivo regulation of plant phospholipid : steryl glucoside ATase by natural lipid microenvironment.

\section{Acknowledgements}

This work was supported in part by research grant BW 1485/33/2000 from Faculty of Biology, Warsaw University, awarded to A.P.

\section{REFERENCES}

Baisted DJ (1978) Steryl glucoside and acyl glucoside biosynthesis in maturing pea seeds. Phytochemistry 17: $435-438$.

Catz DS, Tandecarz JS, Cardini CE (1985) UDP-glucose: sterol glucosyltransferase and a steryl glucoside acyltransferase activity in amyloplast membranes from potato tubers. J Exp Bot 36: 602-609.

Chaudhuri PS, Chaudhuri D, Nanda DK, Achari B, Bhattacharaya D, Saha C (1996) Chemical nature of earthworm repellent factor in the plant (Polygonum hydropiper L.) extract. Ind J Exp Biol 34: 277-278.

Eichenberger W, Siegrist HP (1975) Steryl glycoside acyltransferase from carrots. FEBS Lett 52: 153-156.

Forsee WT, Valkovich G, Elbein AD (1976) Acylation of steryl glucosides by phospholipids. solubilization and properties of the acyl transferase. Arch Biochem Biophys 172: $410-418$.

Galliard T (1968) Aspects of lipid metabolism in higher plants. I. identification and quantitative determination of the lipids in potato tubers. Phytochemistry 7: 19071914.

Guevara A, Lim-Sylianco C, Dayrit F, Finch P (1990) Antimutagens from Momordica charantia. Mutat Res 230: 121-126.

Heinz E, Dieler HP, Rullköter J (1975) Enzymatic acylation of steryl glucoside. Z Pflanzenphysiol 75: 78-87.

Janiszowska W, Wiłkomirski B, Kasprzyk Z (1980) Synthesis of oleanolic acid 3-O-monoglucoside. Pol J Chem 54: 2147-2152.

Kalinowska M, Wojciechowski ZA (1986) Enzymatic synthesis of nuatigenin 3 $\beta$-D-glucoside in oat (Avena sativa L.) leaves. Phytochemistry 25: 2525-2529.

Khan RA, Singh AK, Agrawal PK (1997) Sitosterol sucroside from the suckers of Mentha arvensis. Phytochemistry 45: 1295-1296.

Kintia PK, Wojciechowski ZA (1974) Free and bound sterols in seedlings of Cucumis sativus. Phytochemistry 13: 2235-2238.

Kiriakidis S, Stathi S, Jha HC, Hartmann R, Egge H (1997) Fatty acid esters of sitosterol-3- $\beta$-glucoside from soybeans and tempe (fermented soybeans) as antiproliferative substances. J Clin Biochem Nutr 22: 139-147.

Kiribuchi T, Yasimatsu N, Funahashi S (1967) Synthesis of 6-O-palmitoyl- $\beta$-D-glucosyl- $\beta$-sitosterol. Agric Biol Chem 31: 1244-1246.
Kojima M, Ohnishi M, Ito S, Fujino Y (1989) Characterization of acylmono-, mono-, di-, tri- and tetraglycosylsterol and saponin in adzuki bean (Vigna angularis) seeds. Lipids 24: 849-856.

Lepage M (1964) Isolation and characterization of an esterified form of steryl glucoside. J Lipid Res 5: 587-592.

McDonald RE, Miller WR, McCollum TG (2000) Canopy position and heat treatments influence gamma-irradiation-induced changes in phenylpropanoid metabolism in grapefruit. J Am Soc Hort Sci 125: 364-369.

Misiak M, Kalinowska M, Wojciechowski ZA (1991) Characterization of acyllipid: sterol glucoside acyltransferase from oat (Avena sativa L.) seedlings. Acta Biochim Polon 38: $43-45$.

Moreau RA, Powell MJ, Whitaker BD, Bailey BA, Anderson JD (1994) Xylanase treatment of plant cell induces glycosylation and fatty acylation of phytosterols. Physiol Plant 91: 575-580.

Palta JP, Whitaker BD, Weiss LS (1993) Plasma membrane lipids associated with genetic variability in freezing tolerance and cold acclimation of Solanum species. Plant Physiol 103: 793-803.

Peaud-Lenoel C (1972) D-Glucosylation des phytosterols et acylation des steryl-D-glucosides en presence d'enzymes de plantes. Carbohyd Res 24: 247-262.

Picchioni GA, Watada AE, Whitaker BD, Reyes A (1996) Calcium delays senescence-related membrane lipid changes and increases net synthesis of membrane lipid components in shredded carrots. Postharvest Biol Technol 9: 235-245.

Picchioni GA, Watada AE, Conway WS, Whitaker BD, Sams CE (1998) Postharvest calcium infiltration delays membrane lipid catabolism in apple fruit. J Agric Food Chem 46: 2452-2457.

Potocka A, Zimowski J (2008) Metabolism of conjugated sterols in eggplant. Part 1. UDP-glucose : sterol glucosyltransferase. Acta Biochim Polon 55: 127-134.

Quartacci MF, Cosi E, Navari-Izzo F (2001) Lipids and $\mathrm{NADPH}$-dependent superoxide production in plasma membrane vesicles from roots of wheat grown under copper deficiency or excess. J Exp Bot 52: 77-84.

Staphylakis K, Gegiou D (1985) Free, esterified and glucosidic sterols in cocoa butter. Lipids 20: 723-728.

Whitaker BD, Lee EH, Rowland RA (1990) EDU and ozone protection: foliar glycerolipids and steryl lipids in snapbean exposed to $\mathrm{O}_{3}$. Physiol Plant 80: 286-293.

Wojciechowski ZA (1991) Biochemistry of phytosterols conjugates. In Physiology and Biochemistry of Sterols. Patterson GW, Nes WD, eds, pp 361-393, AOCS, Champaign, Il.

Wojciechowski ZA, Zimowski J (1975) Acyl composition and biosynthesis of acylated steryl glucosides in Calendula officinalis. Biochim Biophys Acta 398: 111-117.

Yamaguchi M, Kasamo K (2001) Modulation in the activity of purified tonoplast $\mathrm{H}^{+}$-ATP-ase by tonoplast glycolipids prepared from cultured rice (Oryza sativa L. var. Boro) cells. Plant Cell Physiol 42: 516-523.

Zimowski J (1991) The occurrence of a glucosyltransferase specific for solanidine in potato plants. Phytochemistry 30: 1187-1193.

Zimowski JG, Wojciechowski ZA (1983) Enzymatic synthesis of steryl 6'-O-acyl- $\beta$-D-glucosides in Sinapis alba and some others plants. Phytochemistry 22: 2175-2178.

Żelazny AM, Shaish A, Pick U (1995) Plasma membrane sterols are essential for sensing osmotic changes in halotolerant alga Dunaliella. Plant Physiol 109: 1395-1403. 